\title{
Acute closure of the left anterior descending artery in a young patient with suspicion of past Kawasaki disease during childhood - A case study
}

\author{
Oleszczak-Kostyra M, Mazur-Jax A* and Dąbrowski M \\ Cardiology Clinic, Bielanski Hospital, Warsaw, Poland
}

\begin{abstract}
Kawasaki disease (KD) is a rare and self-limiting inflammatory disease of medium size blood vessels (mostly of coronary arteries) of unknown etiology. It develops mostly in infants and young children. Its diagnosis is based on typical clinical signs, including: fever, rash, cervical lymphadenopathy, erythema on hands and feet. The inflammatory process may lead to development of aneurysms of coronary arteries and to early atherosclerosis, resulting in acute coronary syndromes in adult patients. We present the case of a 40-years-old male probably post KD in childhood, admitted because of myocardial infarction with ST elevation (STEMI) of the anterior wall, in whom coronarography demonstrated obstruction of LAD and aneurysm of its proximal section. After an unsuccessful angioplasty the patient was qualified for coronary-aortic bypass grafting (CABG) of obstructed LAD, using the MITCAB method. Acute coronary syndromes in patients with history of Kawasaki disease during childhood are described in case reports only, and absence of any clear guidelines makes the analysis of individual cases highly valuable.
\end{abstract}

\section{Case report}

Kawasaki disease $(\mathrm{KD})$ is an acute, self-limiting inflammation of blood vessels, occurring mostly in infants and young babies, but leaving its mark on further life of an adult [1]. During the acute phase it is manifested with high fever, dermatitis and mucositis, and cervical lymphadenopathy [2]. KD involves mostly coronary arteries, and its late complications may also develop in adult patients in form of acute coronary syndromes [1].

We present the case of a 40-years-old male, irregularly treated for arterial hypertension, with history of nicotinism, with no burden of family history of cardiovascular problems, admitted to the Cardiology Clinic because of STEMI of the anterior wall, in the 6th hour after onset of pain. The patient for the first time in his life experienced a typical pain in the chest, lasting for two hours, with accompanying vegetative symptoms: anxiety, weakness and increased perspiration. At admission the patient was in overall relatively good condition, with sufficient circulation and respiration, with increased blood pressure of 160/100 mmHg, SO2 98\%; in ECG - steady sinus rhythm 74 bpm, QS complexes in V1-V4, with ST elevation by $2 \mathrm{~mm}$ in V2-V4. Markers of myocardial necrosis were increased: TnT $2.17 \mathrm{ng} / \mathrm{mL}$ (normal range up to $0.014 \mathrm{ng} / \mathrm{mL}$ ), CKMB $138 \mathrm{ng} / \mathrm{dL}$ (normal range up to $4.87 \mathrm{ng} / \mathrm{dL}$ ). Coronarography performed in urgent mode demonstrated obstructed left anterior descending artery (LAD) and aneurysm of its proximal segment (Figure 1). In the right coronary artery (RCA) an ectasia was observed, involving its third segment (Figure 2). Restoration of patency of LAD was attempted, but was unsuccessful. Conservative treatment of acute coronary syndrome was implemented, according to the typical scheme. Considering long time passed since the onset of pain, a fibrinolytic treatment was not used. Control echo of the heart performed at rest demonstrated apical hypokinesia of the inferior wall, anterior wall and interventricular septum; the global ejection fraction was $52 \%$. On the 8 th day of hospitalisation the patient had exercise

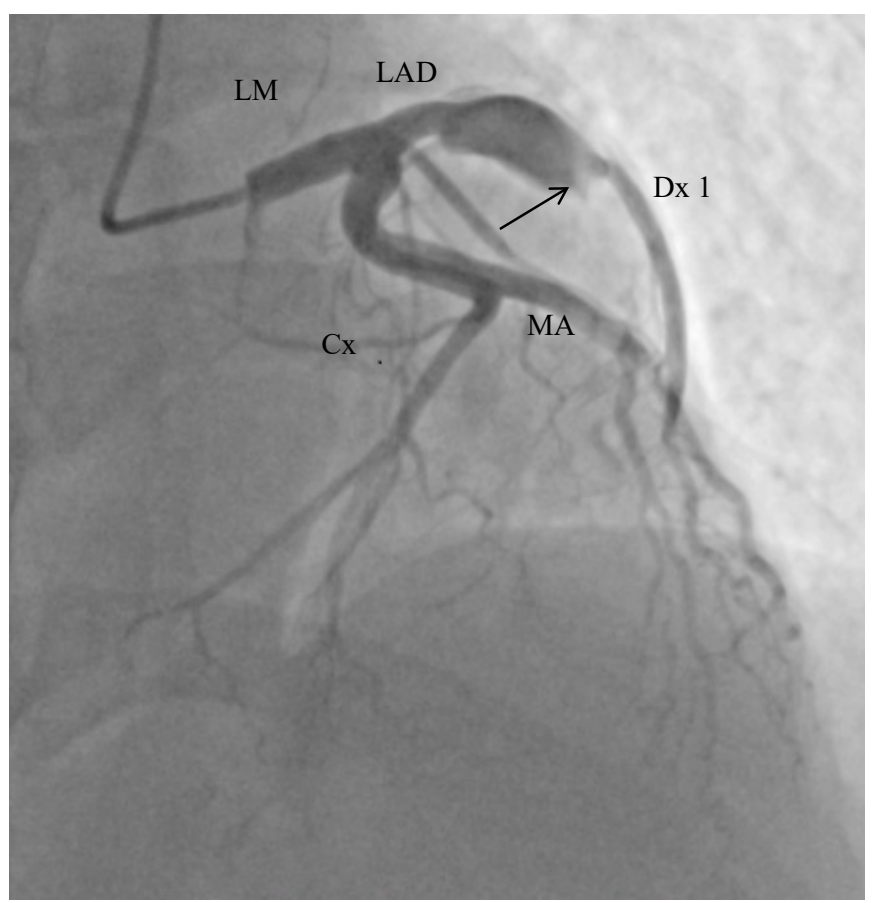

Figure 1. Left coronary artery ( LCA). The aneurysm can be seen in the proximal segment of left anterior descending artery (LAD); the distal part is occluded. ( the arrow shows the amputation of LAD). Cx - Circumflex artery, MA - left marginal artery, Dx- 1st left diagonal artery

${ }^{\star}$ Correspondence to: Mazur-Jax A, Cardiology Clinic, Bielanski Hospital, Warsaw, Poland, E-mail: uskamazur@gmail.com

Received: February 11, 2019; Accepted: February 18, 2019; Published: February 20, 2019 


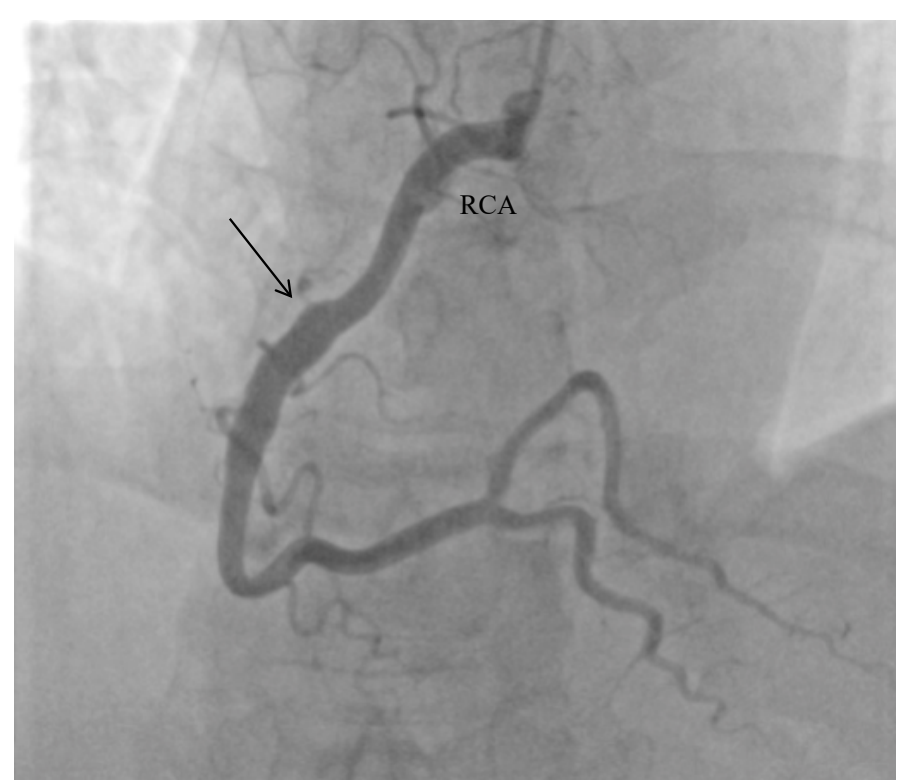

Figure 2. Right coronary artery ( RCA). The arrow shows ectasia of the third segment

echocardiography performed, demonstrating improved contractility of hypokinetic segments of the left ventricle. The patient was qualified for aortic-coronary bypass grafting of the closed LAD, using the MIDCAB method.

Based on the medical history obtained from the patient's mother, a suspicion of Kawasaki disease was made. When the patient was 2 years old, he was hospitalised twice for several weeks in a Pediatric Ward, because of high fever (over 40 degrees Celsius) of unclear origin, with rash and cervical lymphadenopathy. Medical files regarding those incidents could not be accessed.

\section{Discussion}

Kawasaki disease is an acute inflammation of blood vessels, mostly of intermediate size $[1,3]$, and predominantly of coronary arteries. From the point of view of histopathology it is characterised by infiltration of neutrophils extending from the lumen towards the adventitia, with accompanying extensive necrosis of all layers [3]. Progressive narrowing of lumen of the involved artery is a result of its active remodelling, with accompanying proliferation of endothelium and neoangiogenesis $[1,3]$. The endothelium is notably thickened, and contains numerous smooth muscle cells and fibroblasts. Aneurysmal dilation of lumen of the involved vessel is observed proximally to the stenosis. Structure of walls of aneurysms is a consequence of high shear forces. Expression of numerous growth factors was detected in those walls, exerting a direct effect on formation of spindle-shaped aneurysms, characteristic for the disease $[2,3]$.

A cause and pathogenesis of the disease remains obscure [13]. It is suspected, that it is caused by an infectious disease that in individuals possessing some genetic predispositions [2-4] may evoke autoimmune response to bacterial or viral antigens $[2,3]$. KD develops mostly in children between the age of 1 and 3 years, predominantly in males [1]. Initially the disease is manifested with infection of acute and sudden onset, associated with fever not responding to anti-inflammatory treatment, and further development of additional symptoms: conjunctival congestion, red throat, raspberry tongue, cervical lymphadenopathy, skin rash resembling measles or rosacea, hard oedema of hands and feet with accompanying erythema and subsequent exfoliation [1,2]. During the subacute phase of the disease, approx. $20-30 \%$ of patients $[3,4]$ experience involvement of mediumsized blood vessels (most often of coronary arteries), which in extreme cases may cause symptoms of ischaemia or myocardial infarction [1]. The delayed consequence of the inflammatory process within those arteries are aneurysms and a tendency for early development of atherosclerosis. Early treatment with intravenous immunoglobulins (IVIG) may reduce the risk of development of aneurysms. But despite the treatment, ectasia of coronary arteries develops in approx. 5\% of patients $[4,5]$.

The presented patient had had an inflammation of coronary arteries in childhood, which resulted in aneurysmally dilated LAD. CT demonstrated no malformations in large blood vessels. Patients in whom the complication in form of aneurysms developed, are exposed to higher risk of stenosis or acute closure of an artery, resulting in acute coronary syndrome [3].

Adult patients with aneurysms of coronary arteries, unstable angina or myocardial infarction, post Kawasaki disease in childhood, constitute a serious challenge for interventional cardiologists and cardiothoracic surgeons. Angioplasty of coronary arteries in a site below an aneurysm may be difficult, and often proves unsuccessful, as was in the case of the presented patient. There are no clear therapeutic guidelines for that kind of patients [6-10]. In face of absence of guidelines, the analysis of single cases is particularly valuable.

\section{References}

1. Newburger JW, Takahashi M, Burns JC (2016) Kawasaki Disease. J Am Coll Cardiol 67: 1738-1749. [Crossref]

2. Scuccimarri R (2012) Kawasaki Diseas; Pediatric Clinics of North America 59: 425-445.

3. Mandal S, Pande A (2012) Mandal A, Sarkar M, Kahali D,Various coronary artery complications of Kawasaki disease: Series of 5 cases and review of literature $J$ Cardiovasc Dis Res 3: 231-235.

4. Gowin E, Małecka I, Stryczyńska-Kazubska J, Michalak M, Wysocki J, et al. (2016) Cardiac complications in children with Kawasaki disease in our own experience. Kardiologia Polska (Polish Heart Journal) 74: 75-82.

5. Berdej-Szczot E, Malecka-Tendera E, Gawlik T, Firek-Pedras M, Szydlowski L, et al Risk factors of immunoglobulin resistance and coronary complications in children with Kawasaki disease. Kardiol Pol 75: 261-266.

6. Etsuko T, Tadaaki A, Wataru T (2011) Acute coronary syndrome in adult patients with coronary artery lesions caused by Kawasaki disease:review of case reports. Cardiology in the Young 21: 74-82.

7. Okumori M, Tokuno S, Nogami Y, Kann I, Tanaka Y (1995) Treatment of a gian coronary artery aneurysm in an adult with a history of Kawasaki disease by resection and bypass grafting: report of a case. Surg Today 25: 373-377.

8. Kitamura S, Kameda Y, Seki T, Kawachi K, Endo M, et al. (1994) Long-term outcome of myocardial revascularization in patients with Kawasaki coronary artery disease. A multicenter cooperative study. J Thorac Cardiovasc Surg 107: 663-673

9. Codispoti C, Boyd S, Sees D, Conner W (2008) Symptomatic coronary obstruction due to Kawasaki disease in an adult. Ann Thorac Surg 85: 1081-1083. [Crossref]

10. Reinerth G, Konradi D, Rupprecht HJ, Münzel T, Vahl CF (2006) Revascularisation of a giant coronary artery aneurysm in suspected incomplete Kawasaki-disease. Thorac Cardiovasc Surg 54: 358-359.

11. Dohmen G, Dahm M, Elsner M, Kasper W, Oelert H (2000) Coronary artery bypass grafting in adult coronary artery disease due to suspected Kawasaki disease in childhood. Ann Thorac Surg 70: 1704-1706.

Copyright: C2019 Oleszczak-Kostyra M. This is an open-access article distributed under the terms of the Creative Commons Attribution License, which permits unrestricted use, distribution, and reproduction in any medium, provided the original author and source are credited. 